\title{
mediRobbi: An Interactive Companion for Pediatric Patients during Hospital Visit
}

\author{
Szu-Chia Lu, Nicole Blackwell, and Ellen Yi-Luen Do \\ Georgia Institute of Technology, GVU Center \\ Atlanta, GA 30318, USA \\ \{sj.today, nblackwell3, ellendo\} @gatech. edu
}

\begin{abstract}
Young children often feel terribly anxious while visiting a doctor. We designed mediRobbi, an interactive robotic companion, to help pediatric patients feel more relaxed and comfortable in hospital visits. mediRobbi can guide and accompany the pediatric patients through their medical procedures. The sensors and servomotors enable mediRobbi to respond to its environmental inputs and the reactions from young children as well. The ultimate goal of this study is to transform an intimidating medical situation into a joyful adventure game for the pediatric patients.
\end{abstract}

Keywords: Pediatric care, robotics, Children behavior.

\section{Introduction}

While some young children are confident about visiting the pediatrician or dentist, others are filled with dread and anxiety at the thought of the experience. This fearfilled event can be unpleasant for parents and children alike. The visit can quickly become a battle before, during or even after the exam. The reason young children experience this anxiety is the fear of the unknown, which is often more frightening than the reality. Under these circumstances, a tool to put the child's mind at ease would be greatly appreciated. An explanation, including what to expect from the doctor or nurse, would be beneficial in helping to mentally prepare the child for the process [1].

However, an explanation alone could not eliminate anxiety once the medical procedure begins. If an uncomfortable step needs to be performed (a simple throat swab, a scheduled vaccination, a weight/height recording, or a blood pressure check), the child needs to feel safe and secure about the impending proceedings.

For this reason, having a comforting object with them during the doctor's visit can provide further relief. Children can usually benefit from having a comforting object with them during their physical exams [2][3]. These friendly objects can help a child remain calm. Young children find comfort in a stuffed animal or a security blanket; older children may choose a favorite article of clothing or a book. These comforting objects play the role of a sharing companion, a consolatory pet or a brave friend that helps the child remain confident while redirecting the attention to something else.

An early report described the benefits of Animal-Assisted Therapy (AAT) [4], in which the animal companion would serve as a communication link, and provide the 
child, even the one with autism, with a sense of security in the therapy setting and perceived shortened therapy process. For example, Boris Levinson, M.D., a Canadian child psychiatrist, included his dog in therapy sessions [5]. However, not all pediatric hospitals can afford to have animal companion for each child during their hospital visit. Therefore, we are interested in developing a robotic pet to serve this companion function.

In this project, we utilized the Lego Mindstorms' building components to design and program an interactive robotic pet called mediRobbi. Equipped with various types of sensors, mediRobbi can detect light levels, distances of other objects in front of the robot, sounds from the surroundings, and touches from people or other objects. These sensors enable mediRobbi to receive signals from the pediatric patient, doctor, or even the environment. Signals such as colors or symbols on a medicine label, distance of the medical equipment, sound from the young patients, or behaviors of the doctor can all be perceived by the mediRobbi robot. Most importantly, the mediRobbi can generate motor, visual, or audible feedback in response to those inputs through its servomotors. Through the companionship of mediRobbi, the ultimate goal of this project is to help the pediatric patients transform their intimidating medical experiences into a joyful game play.

\section{Related Work}

\subsection{Comforting Objects during Hospital Visit}

Examples of comforting objects in hospitals are abundant. For example, the Sons of the American Legion in Hays initiated the "comforting kit" program to ease the intimidating experience of the pediatric patients who spend the night at Hays Medical Center [6]. They send out the comforting kit called "a Josh and Friends kit". The package includes an adorable plush puppy "Josh" and a book, named "I'll Be OK"[7]. Both the puppet and the storybook were packaged in a doghouse that is ready for those young patients who are visiting the hospital for the first time. The director of Women and Children Nursing Services at Hays Med, Celeste Gray, stated that this comforting kit has helped make the hospital more of an adventure for the child and possibly relieve some of the stress.

Meanwhile, another institute, the Emerson Hospital, in Massachusetts also distributed bags of toys and books, called Coping Kits, to comfort their pediatric patients and to support the parents during visits to their hospital's Emergency Department [8]. The idea of this Coping Kits was developed by the hospital's Pediatric Intervention Team, including a Child Life Specialist, a registered nurse and the team's nurse coordinator. The content for each Coping kits varies for children by age and color coded by gender. Every kit contains five tools for five different coping behaviors for children: 1) distract from the stressful exam process, 2) encourage relaxation and deep breathing, 3) provide comfort, 4) require focusing on an object, and 5) encourage communication and self-expression.

The above examples of hospital practices demonstrate the importance of a companion or a comforting object for young patients to alleviate the stress during the medical process and to bridge the communication between the medical staff and the 
children. However, these coping and comforting kits cannot produce real-time responsive support. The toys or storybooks can be beneficial to prepare the pediatric patients for their hospital visits or serve as great distractions for children to focus their attention on something other than the dreadful medical procedures. However, they cannot respond to those young patients' behaviors or reactions. Young children might get bored very easily with unresponsive objects. Therefore, we designed mediRobbi to be interactive and responsive.

\subsection{Human-Companion Animal Interaction}

Animal-Assisted Therapy (AAT) is another therapeutic aspect about interaction between the young children and their pets to provide strong emotional bonds that serves as a source of support to the pet owners [4][5][9]. We believe our young patients can benefit from this kind of support. Several psychology studies validated that pets could contribute to the development of 1) a child's basic sense of trust through the pet's constancy, security, reliability, love and affection; 2) a sense of autonomy through the pet's serving as an active playmate and encouraging patience and self-control; 3) a sense of industry through the pet's trainability and response to the child's basic commands; and 4) a sense of identity through the pet's serving as a companion, and providing social and emotional support [10][11][12]. These four senses are quite important either for just a short-term one-time hospital visit or a longterm chronic treatment for pediatric patients.

These Animal-Assisted Therapy studies reported that responsive pets served as an excellent communication link that provides the young patients with a sense of security in the hospital setting. These responsive companions quickened the medical process as well.

\section{Initial Prototype of mediRobbi Using Lego Mindstorms}

For a proof-of-concept prototype of this interactive companion, Lego Mindstorms NXT was used to build this interactive robot. Lego Mindstorms is a line of programmable robotics construction toy, manufactured by the Lego Group [13]. It comes in a package containing many pieces including sensors and cables. These input sensors including a touch sensor that enables the robot to feel and react to its environment, a sound sensor so that the robot can hear and react to sound, a light sensor to detect light and color for the robot, an ultrasonic sensor providing the robot the ability to see, measure distance to an object, and react to movement, and, at last, three interactive servo motors that ensure the robot movement with precision. With the help of these sensors, the robot will be able to receive signals from the pediatric patient, doctor, or even the environment.

We followed the building instruction in Lego's Robo Center and built a rapid prototype of an interactive pet by using the input sensors and the building bricks (See Figure 1). It was equipped with all five types of sensors, a touch sensor, a sound sensor, a light sensor, an ultrasonic sensor, a light sensor and three servomotors. The robot even has hands to hug or grab small objects. 
Then, the robot was programmed into sets of reaction orders through the Lego Programming Palette (See Figure 2). The programming palette contains all of the programming blocks a creator will need to create programs. Each programming block determines how the robot will act or react. By combining blocks in sequence, a creator can quickly create programs that will make the robot come to life.

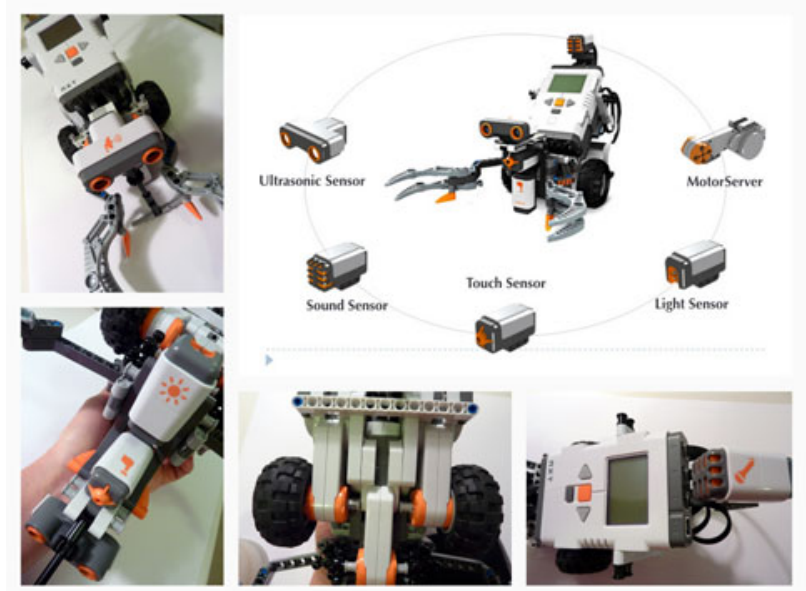

Fig. 1. In top-right, the middle model is the interactive pet we built. Around the interactive pet are the input sensors used in the model.

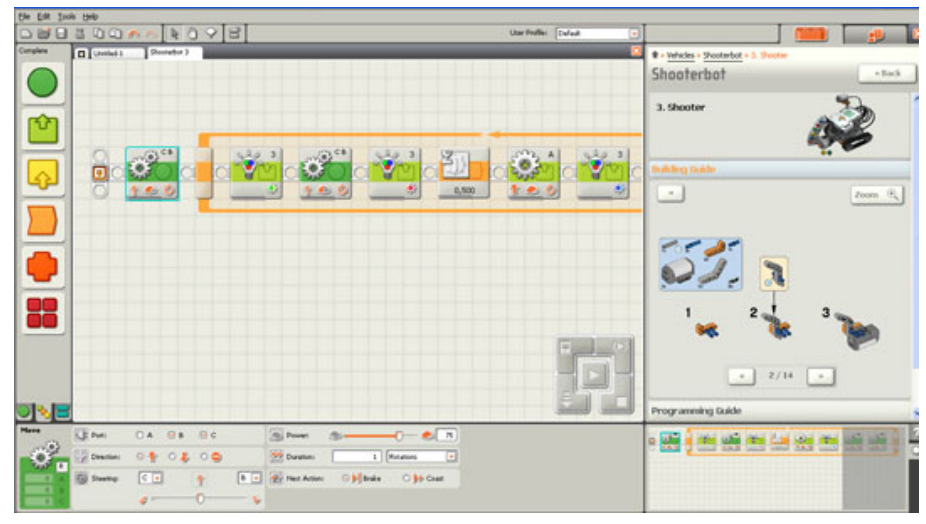

Fig. 2. The Lego Mindstorms programming palette. Using the drag-and-drop command block to design the interaction for the robot.

There are five interactions sets for the pet robot. These interaction sets were designed to play the game Stop-and-Go, which is a board game explaining allergy related information to the children.

The first interaction set is activation. Pushing the stick to press down the touch sensor, which is underneath the head of the robot, can activate the robot. After putting 
the robot on the map and activating it, the robot will follow the order of next interaction set to follow the black route on the map. This activation feature is critical in the play of the adventure board game, and in the next phase, in leading the pediatric patients through their examination as well.

The second and the third interaction sets were accomplished by using the light sensor equipped facing down to read the black track or red spot on the white map. The light sensor can detect the degree of brightness, and adjust the motor movement accordingly.

The fourth design of interaction is that the robot will reactivate after stopping at a red spot by a loud sound or voice, such as clapping or shouting out. This interaction can be triggered by the sound detected by its sound sensor.

The last interaction feature is visual and audible feedback from the robot. When the robot reaches the home station on the map or achieves a task goal, it will trigger the hooray sound effect download to the robot, and a simple dancing movement of clapping its hands.

\section{Experiment Design}

In order to examine whether the children would enjoy the company of their new friend during a stressful situation of a hospital visit, we are conducting a paired interview of children and their parents and a field observation study of children in the hospital.

\subsection{Paired Interview}

The paired interview involves young children in the age range of 3 to 7 and their parents. The interview includes questions about the experience of hospital visit of young children and how their parents prepare them mentally or physically before or during the hospital visits.

A simple questionnaire and consent form will be conducted before the experiment. The questionnaire will collect the following information:

1. About the parents. Age range of the parents, how many children (in the age range of 3-7) within the family, frequency of hospital visit, approximate duration in one regular hospital visit (including the waiting time), how they prepare the child before/during the hospital visit, and specific reaction (if any) during the hospital visit.

2. About the child. Age, gender, how they feel when visiting a doctor and why, what they particularly like/hate during the hospital visit, would they like to have a companion during the hospital visit?

These data were collected through interview in person or via phone, and stored in electronic format in a secured online database.

\subsection{Field Observation of Children Behavior}

The field observation was conducted in a pseudo hospital environment. We utilized the empty lab space into a pediatric examine room and a children waiting/playing area. There are three phases for the field observation: $1^{\text {st }}$ phase of Introduction, $2^{\text {nd }}$ 
phase of Familiarization and $3^{\text {rd }}$ phase of Real Examination. Each phase has its purpose for young participants to know the companion, mediRobbi, to get familiar how mediRobbi would react to their behavior or orders, and then to start the exam process after they feel comfortable being with their companion.

First Phase - Stage of Introduction. The purpose of this phase is to present the mediRobbi to our young participants and to start building the trusting connection between them and the companion. In the Introduction phase, the young participants were asked to choose the name and gender of their companion from an interactive webpage (see Figure 3). Then, the young patients decided how they want to decorate or dress their companion by choosing the clothing pattern from the same website

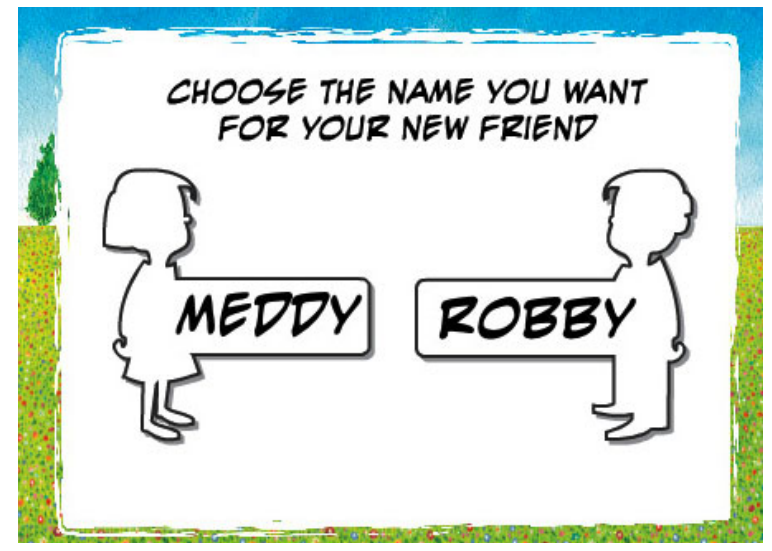

Fig. 3. Snapshot of the website for the children to choose name and gender for their new companion

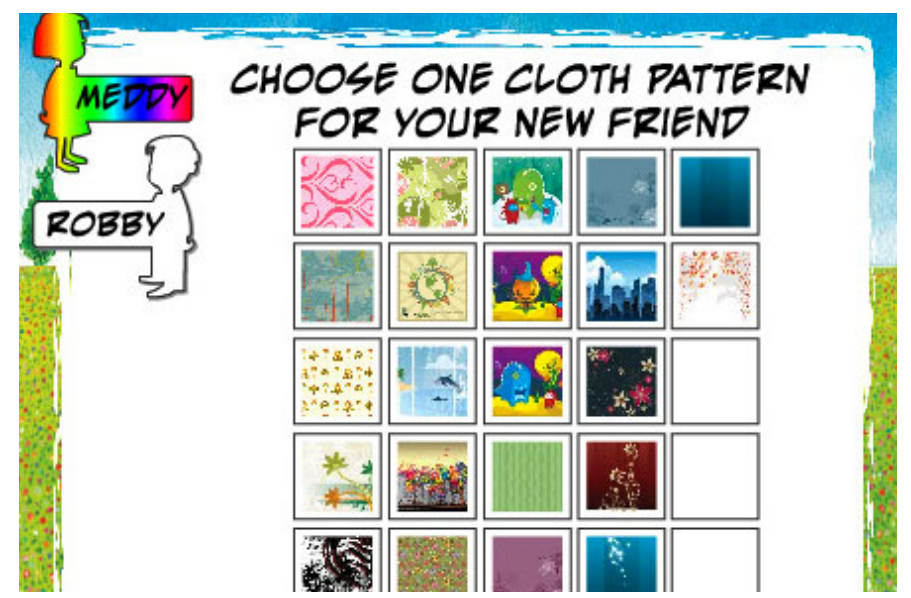

Fig. 4. This is the snapshot of the second step for children to choose one of decoration pattern for their new companion 
(see Figure 4). After the choosing step, the premade origami shirt or dresses (see Figure 5) was attached to mediRobbi and present the personalized companion to the young participant.

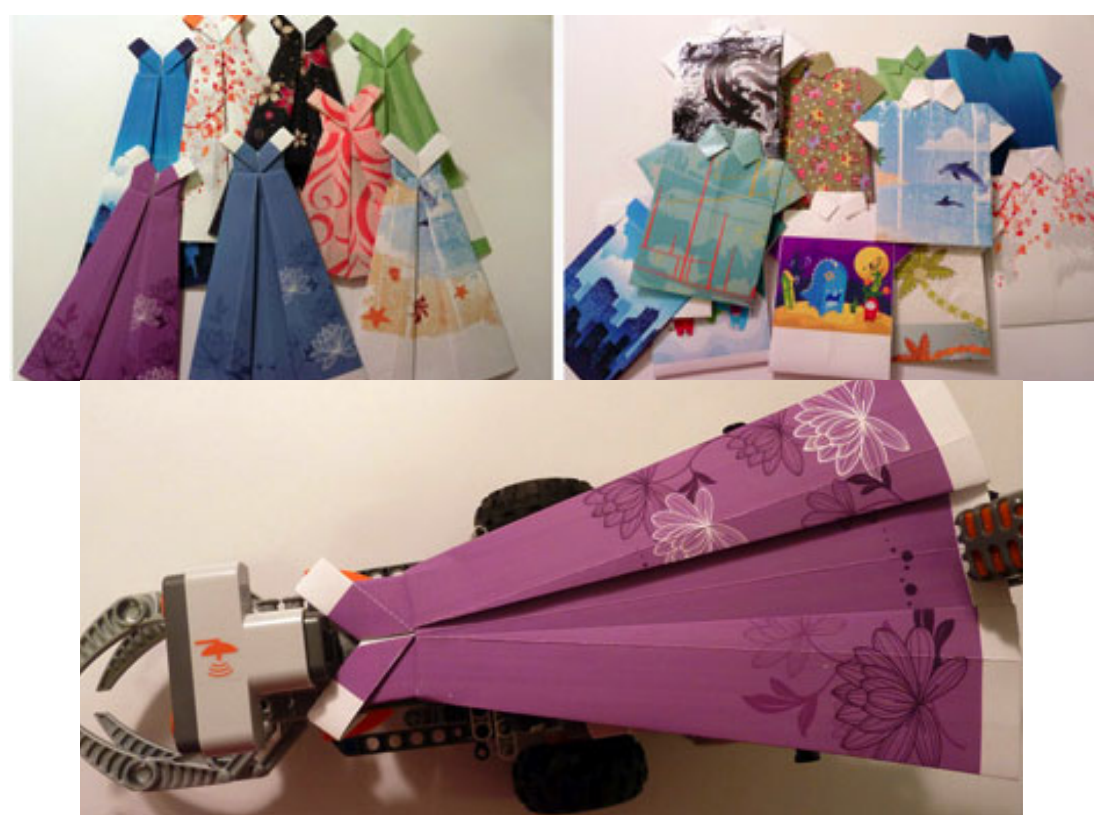

Fig. 5. An origami dress is attached to the interactive companion

Second Phase - Stage of Familiarization. The second phase is for the young patients to familiar themselves with their new playmate by running their mediRobbi on an educational allergy board game, called Stop-and-Go, a 36"x48" carpet-sized map. The main purpose of this game is to increase the sense of ownership of the young participants and to build the trusting relationship between the young participant and the mediRobbi through the leading-following process. During the gameplay, the children would also learn how mediRobbi could act or react, and how they can control the behavior of mediRobbi.

We designed a game, the Stop-and-Go Food, and a game map on a large, colorful, configurable map (see figure 6) for kids to use along with the robot. The game map creates a playground space for the young patient and his/her new friend, the mediRobbi. The robot serves as an encouraging interactive companion to facilitate play in the game space. This Stop-and-Go game introduces youngsters to a typical peanut allergy. While the robot travels along the predetermined path, it will stop each time at a decision point of Stop (peanut-related) food item. Each decision point has a rotating wheel that allows the child to select a given food item to present to the robot to resume motion. If the child chose the item that does not contain peanuts, the path would be connected and the robot can continue to move. If the choice is a food item that could cause allergy reaction, the path segment would be of red color and therefore the robot would not move. The goal of the game is to help the mediRobbi robot properly negotiate the food allergy constraints and find its way home. 


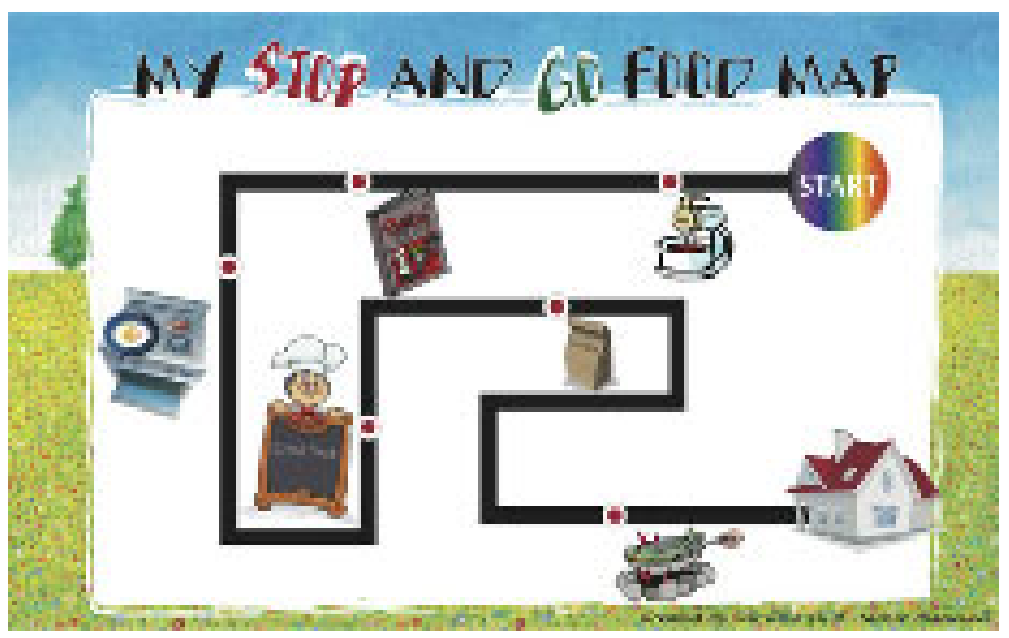

Fig. 6. The game map was designed for the board game of the Stop-And-Go Game
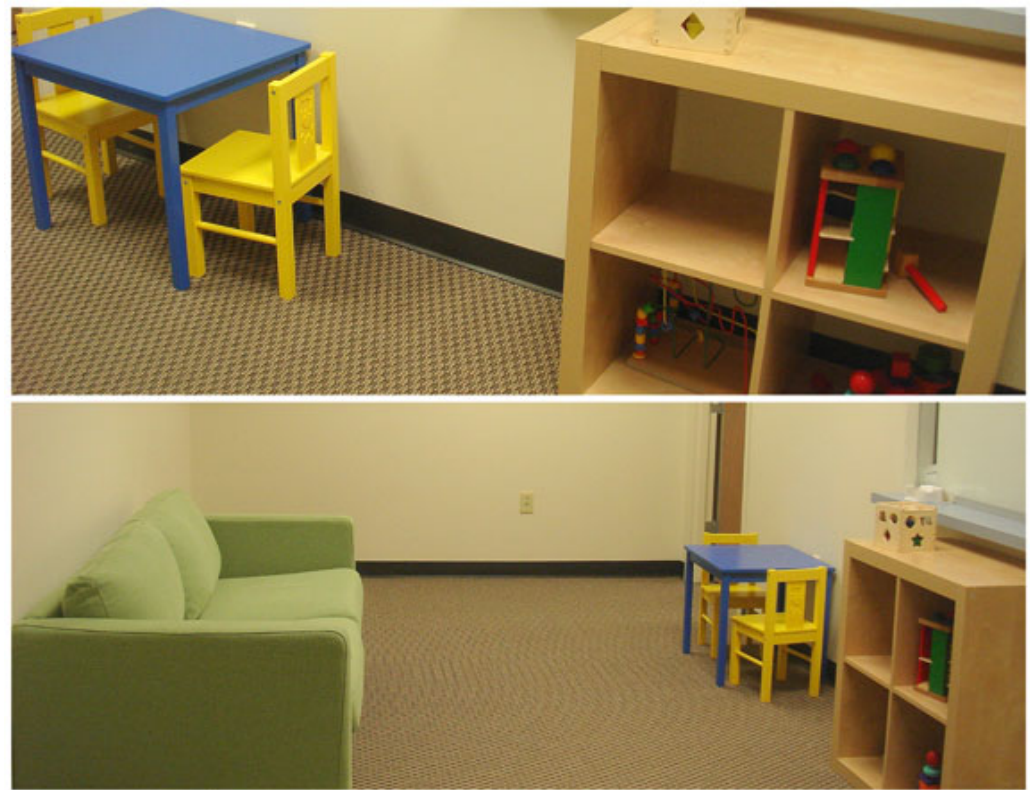

Fig. 7. The pseudo children playing/waiting room inside the Child Behavior Lab

During this familiarization stage, the Stop-and-Go game requires the child to familiar with the robot by giving orders and to learn what feedback the robot would react. The interaction requires the motor skills of the robot pet to move along the path. The child learns that mediRobbi is capable of motion. The robot uses light sensor to 
differentiate the black path from any motion-halting white spaces or red spots. The child learns that mediRobbi can be controlled to resume the motion. In short, the children can learn how to interact with the mediRobbi in this stage.

Third Phase - Stage of Real Examination. In the third phase, the participant started their pseudo examination process with our fake doctor in a pseudo hospital environment. We utilized the space in Child Behavior Lab where our young participants proceeded the first two phases in the playroom (see Figure 7) and the third phase in another divided space next to this playroom.

Instead of leading mediRobbi back home, the leading and following roles were reversed. The mediRobbi served as a guardian for the children and showed the young patient how long and how many steps of the examination process would be. The mediRobbi walked through a pre-designed channel of route visualizing the length of the whole visit. That the young participant can get a general idea of how long the exam would be. Also, their new friendly companion, mediRobbi, was there and accompanied them through the whole process.

\section{Design Challenge and Discussion}

One major challenge encountered during the implementation of Lego Mindstorms is that there's some limitation within the Lego device that we have to adjust our interaction design based on the robot behavior. The other major concern about the Lego Robot is its size and weight. Some of the young children seemed to have trouble holding the robot to bring the robot around. Besides, they really have to be gentle with the robot in order not to break it. This is also difficult for the young children. Future design should adjust the size and weight of the companion so that young children can carry the companion with them easily.

\section{Summary}

By moving through the three-phased process, from introduction phase to real examination, mediRobbi allows the pediatric patient to create a personalized playmate, to engage in a trust relationship with a comforting friend, and to learn about health issues through engaging play. These concepts were considered in the whole interaction design to help young pediatric patients overcome unknown anxiety and fear during their hospital visit.

\section{References}

[1] Rasnake, L., Linscheid, T.: Anxiety Reduction in Children Receiving Medical Care: Developmental Considerations. Journal of Developmental And Behavioral Pediatrics 10(4), 169-175 (1989)

[2] Farnum C.: How to Make Doctors' Visits Easier for Kids (March 24, 2010), http: / /www. suite101.com/content/ how-to-make-doctors-visits-easier-for-kids-a217717 
[3] How to Prepare Your Child for a Hospital Visit, http: / /www.ehow.com/how_3939_prepare-child-hospital.html

[4] Martin, F., Farnum, J.: Animal-Assisted Therapy for Children with Pervasive Developmental Disorders. Western Journal of Nursing Research 24(6), 657-670 (2002)

[5] Levinson, B.M.: The dog as a "co-therapist",? Mental Hygiene 46, 59-65 (1962)

[6] Pediatric Patients at Hays Med Get a Companion, http: //portal.haysmed.com/portal/ page?_pageid=43,67257\&_dad=portal\&_schema=PORTAL

[7] Lange, R. L.: I'll be O.K., First Printing. JoshCo, LLC (1997)

[8] Coping Kits Comfort Children in the Emergency Department, http: / /www . nursezone.com/nursing-news-events/more-features / Coping-Kits-Comfort-Children-in-the-EmergencyDepartment_20482.aspx

[9] Levinson, B.M., Mallon, G.P.: Pet-Oriented Child Psychotherapy, 2nd edn. Charles C. Thomas Publisher (1997)

[10] Robin, M., Bensel, R.T.: Pets and the Socialization of Children. Marriage \& Family Review 8(3), 63 (1985)

[11] Blue, G.F.: The Value of Pets in Children's Lives. Childhood Education 63(2), 84-90 (1986)

[12] Bryant, B.K.: The Richness of the Child-Pet Relationship: A Consideration of Both Benefits and Costs of Pets to Children. Anthrozoos: A Multidisciplinary Journal of The Interactions of People \&\#38; Animals 3(9), 253-261 (1990)

[13] Lego.com Mindstorms, http://mindstorms. lego.com/en-us/Default.aspx 